OPEN ACCESS

Edited by:

Xinran Ma,

East China Normal University, China

Reviewed by:

Farnaz Shamsi,

Joslin Diabetes Center and Harvard Medical School, United States

Fang Hu,

Central South University, China

*Correspondence: Xuemei Tong xuemeitong@shsmu.edu.cn

${ }^{\dagger}$ These authors have contributed equally to this work and share first authorship

Specialty section:

This article was submitted to Cellular Endocrinology, a section of the journal Frontiers in Endocrinology

Received: 15 January 2021 Accepted: 15 March 2021

Published: 31 March 2021

Citation:

Ke H, Luan Y, Wu S, Zhu Y and Tong X (2021) The Role of Mondo Family Transcription Factors in NutrientSensing and Obesity.

Front. Endocrinol. 12:653972. doi: 10.3389/fendo.2021.653972

\section{The Role of Mondo Family Transcription Factors in Nutrient- Sensing and Obesity}

\author{
Huiyi $\mathrm{Ke}^{\dagger}$, Yu Luan ${ }^{\dagger}$, Siming $\mathrm{Wu}^{\dagger}$, Yemin Zhu and Xuemei Tong ${ }^{*}$ \\ Department of Biochemistry and Molecular Cell Biology, Shanghai Key Laboratory for Tumor Microenvironment and \\ Inflammation, Key Laboratory of Cell Differentiation and Apoptosis of Chinese Ministry of Education, Shanghai Jiao Tong \\ University School of Medicine, Shanghai, China
}

In the past several decades obesity has become one of the greatest health burdens worldwide. Diet high in fats and fructose is one of the main causes for the prevalence of metabolic disorders including obesity. Promoting brown or beige adipocyte development and activity is regarded as a potential treatment of obesity. Mondo family transcription factors including MondoA and carbohydrate response element binding protein (ChREBP) are critical for nutrient-sensing in multiple metabolic organs including the skeletal muscle, liver, adipose tissue and pancreas. Under normal nutrient conditions, MondoA and ChREBP contribute to maintaining metabolic homeostasis. When nutrient is overloaded, Mondo family transcription factors directly regulate glucose and lipid metabolism in brown and beige adipocytes or modulate the crosstalk between metabolic organs. In this review, we aim to provide an overview of recent advances in the understanding of MondoA and ChREBP in sensing nutrients and regulating obesity or related pathological conditions.

Keywords: MondoA, ChREBP, nutrient-sensing, obesity, brown and beige adipose tissue

\section{INTRODUCTION}

The epidemic of obesity has emerged as a worldwide public health issue. In 2017, the Global Burden of Disease Study estimated that high body mass index (BMI), one of the leading risk factors, accounted for 4.72 million deaths and 148 million disability-adjusted life-years (DALYs) (1). Excessive caloric intake mainly derived from the high-fat and high-fructose diet is a major cause for obesity (2-4). The urgent need for weight-loss treatments has given rise to multiple attempts to target cellular metabolism and restore systemic energy homeostasis, among which is the strategy of promoting brown and beige adipocyte activity and development. Brown adipocytes are essential for thermogenesis in mammals with their characteristic expression of uncoupling protein-1 (UCP1) in mitochondria, while beige adipocytes are inducible to express thermogenic genes in response to stimulus (5). Enhancing activities of brown and beige adipocytes not only promotes energy expenditure through heat generation, but also enhances glucose metabolism and protects against insulin resistance (6-11), which provides promising therapeutic effects to counteract obesity and related diseases.

The Mondo family transcription factors, comprised of MondoA (also known as MLXIP) and carbohydrate response element binding protein (ChREBP, also named MondoB and MLXIPL), 
belong to the basic helix-loop-helix leucine zipper (bHLH/LZ) family $(12,13)$. Upon binding to their heteromeric partner MLX (Max-like protein X), Mondo and MLX translocate to the nucleus where they bind to carbohydrate response elements (ChoREs) on target gene promoters, and stimulate a transcriptional response to nutrients (12-14). As a structural basis of their nutrient-sensing and responsiveness, the glucosesensing module (GSM) of Mondo proteins consists of a lowglucose inhibitory domain (LID) and a glucose-response activation conserved element (GRACE) (Figure 1). Under basal conditions, GRACE is repressed by the LID domain, which is relieved by alterations in nutrient levels such as the elevation of glucose concentration (15). An isoform of ChREBP, ChREBP $\beta$, lacks the LID domain and is induced by the activation of the canonical isoform ChREBP $\alpha$ (16). Upon activation, MondoA and ChREBP bind to importin- $\alpha$ which mediates their nuclear entry (17), while their nuclear export and cytoplasmic retention are regulated by chromosome region maintenance protein 1 (CRM1) and 14-3-3 proteins (18-20). Though similar in structure, MondoA and ChREBP have distinct tissue distribution patterns, with MondoA predominantly in skeletal muscle and immune cells and ChREBP in liver and adipose tissue $(12,13)$, and our unpublished data.
Initially identified as glucose sensors, Mondo family has more extensive regulatory functions in metabolic homeostasis. Therefore, their role in physiological and pathological conditions has gained growing interest. In this review, we will discuss how MondoA and ChREBP sense and respond to nutrient availability, focusing on the involvement of Mondo family in obesity and related diseases.

\section{NUTRIENT-SENSING BY MONDOA AND CHREBP}

\section{ChREBP in Metabolic Organs}

ChREBP is widely expressed in metabolic organs, predominantly in liver, also in adipose tissues, pancreas, intestine, kidney, relatively low in skeletal muscle (21).

ChREBP is regulated by multiple nutrient molecules, among which glucose and its metabolites are major determinants. In the presence of high glucose, glucose 6-phosphate (G6P), the first intermediate in glycolysis binds to the GRACE domain of ChREBP $(22,23)$. Moreover, xylulose 5-phosphate (Xu5P), the metabolite generated through the pentose phosphate pathway, activates protein phosphatase $2 \mathrm{~A}(\mathrm{PP} 2 \mathrm{~A})$, and the sequential
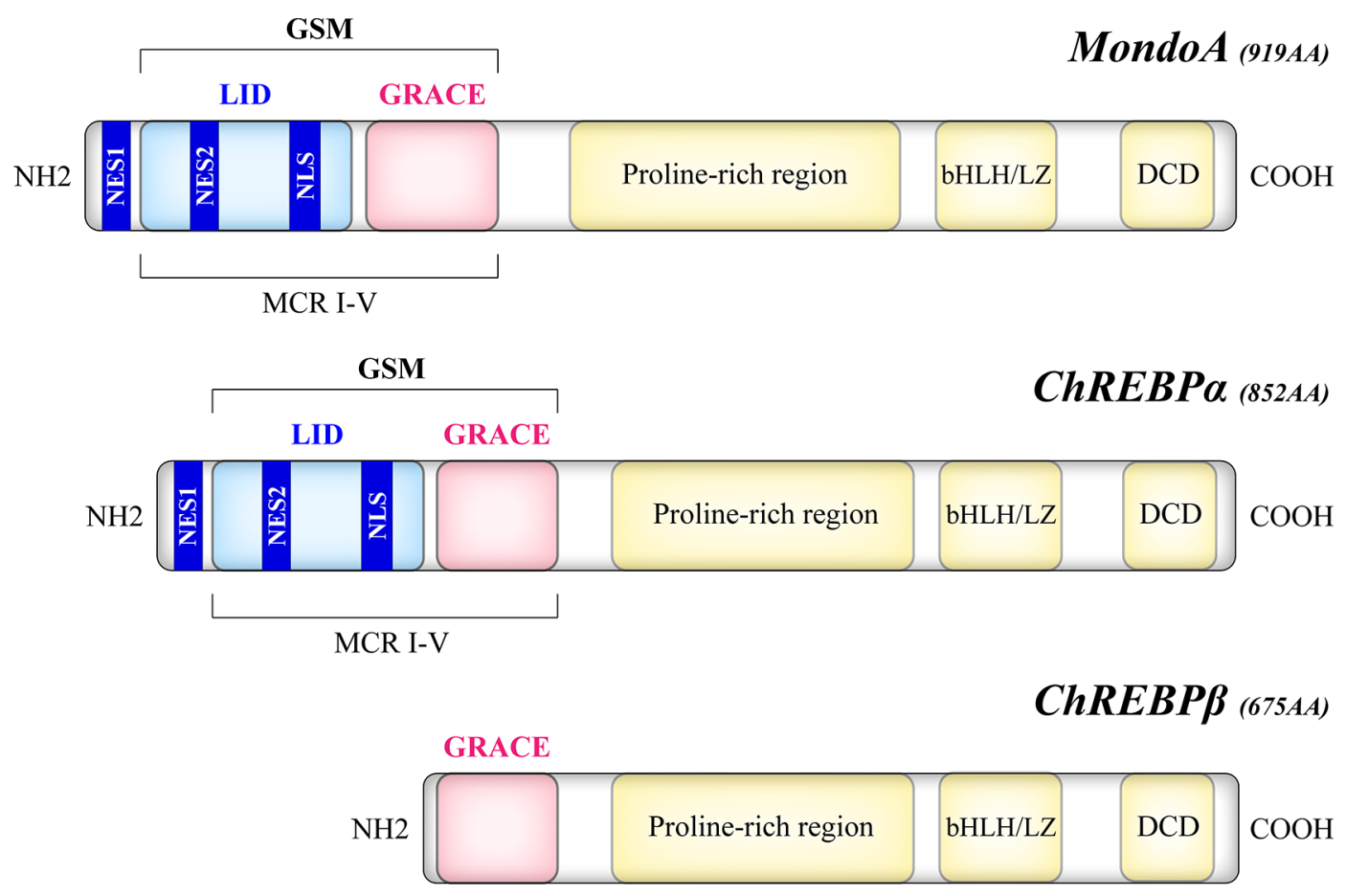

FIGURE 1 | Structural domains of the human Mondo proteins. MondoA and ChREBP contain homologous C-termini, where a bHLH/LZ region and a dimerization and cytoplasmic localization domain (DCD) mediate the heterodimerization process and DNA binding. On the other hand, the N-termini of MondoA and ChREBP determine their glucose responsiveness. The glucose-sensing module (GSM) lies within the N-terminal region of MondoA and ChREBP $\alpha$ and is composed of a lowglucose inhibitory domain (LID) and a glucose-response activation conserved element (GRACE). Compared with the canonical ChREBP $\alpha$ of 852 amino acids, the ChREBP $\beta$ isoform, a product of alternative splicing, is a 675-amino acid protein that lacks the LID domain. The N-terminal region also includes five Mondo conserved regions (MCR I-V), with LID spanning MCR I-IV and GRACE harboring MCR V. Two nuclear export signals (NES1, NES2) correspond to the binding site of CRM1, while a nuclear localization signal (NLS) allows the interaction with importin- $\alpha$. 
dephosphorylation of several residues activates ChREBP (24). Furthermore, fructose-2,6-bisphosphate (F2,6-BP) derived from fructose-6-phosphate (F6P) has been identified as another signaling metabolite responsible for glucose-induced recruitment of ChREBP to its target genes (25). On the other hand, when glucose is limited, branched chain keto-amino acids (BCKA) and fatty acids (FA) inhibit ChREBP activity $(26,27)$ (Figure 2).

ChREBP regulates many enzyme genes in glycolysis and lipogenesis, including liver type pyruvate kinase (L-PK), acetylCoA carboxylase (ACC), fatty acid synthase (FAS), ATP-citrate lyase (ACLY), stearoyl-CoA desaturase-1 (SCD1) and glycerol-3phosphate dehydrogenase $(\mathrm{GPDH})(28-30)$. In addition, ChREBP may control very low-density lipoprotein (VLDL) export by regulating microsomal triglyceride transfer protein (MTTP) transcription (31) (Figure 3).

Mouse models with knockout or overexpression of the ChREBP gene provide direct evidence for its role in glucose and lipid metabolism (Table 1). ChREBP global knockout mice show down-regulated pyruvate production and inhibited glycolysis, with lower mRNA levels of ACC, FAS, ACLY and SCD1 in liver than wild-type mice, leading to a significant decrease in lipids converted from glucose (32). ChREBP liverspecific knockout mice showed dysregulation of glucose response and impaired glucose homeostasis (34). Moreover, adenoviral overexpression of ChREBP caused higher liver triglyceride contents with increased FAS and ACC expression $(35,46)$. It is now believed that ChREBP and sterol regulatory element binding protein-1c (SREBP-1c) play a synergistic role in the regulation of lipogenesis in liver (47). Moreover, the expression of the ChREBP $\beta$ isoform is associated with the respective increase and repression of branched chain alpha-keto acid dehydrogenase kinase (BDK) and protein phosphatase $\mathrm{Mg}^{2}$ ${ }^{+} / \mathrm{Mn}^{2+}$-dependent $1 \mathrm{~K}$ (PPM1K) transcript levels in liver (48). In addition, the transcription of fibroblast growth factor 21 (FGF21) is regulated by ChREBP (49). FGF21 is involved in energy metabolism by regulating carbohydrate intake (50). Fructose ingestion increases FGF21 production in a ChREBPdependent manner while FGF21 knockout attenuates ChREBP expression and de novo lipogenesis following fructose consumption, indicating that ChREBP and FGF21 constitute a signaling axis which mediates an adaptive hepatic metabolic response to fructose ingestion (51).

\section{MondoA in Metabolic Organs}

As another transcriptional biosensor of intracellular glucose concentration, MondoA contributes to more than $75 \%$ of glucose-induced transcription signature in HA1ER epithelial

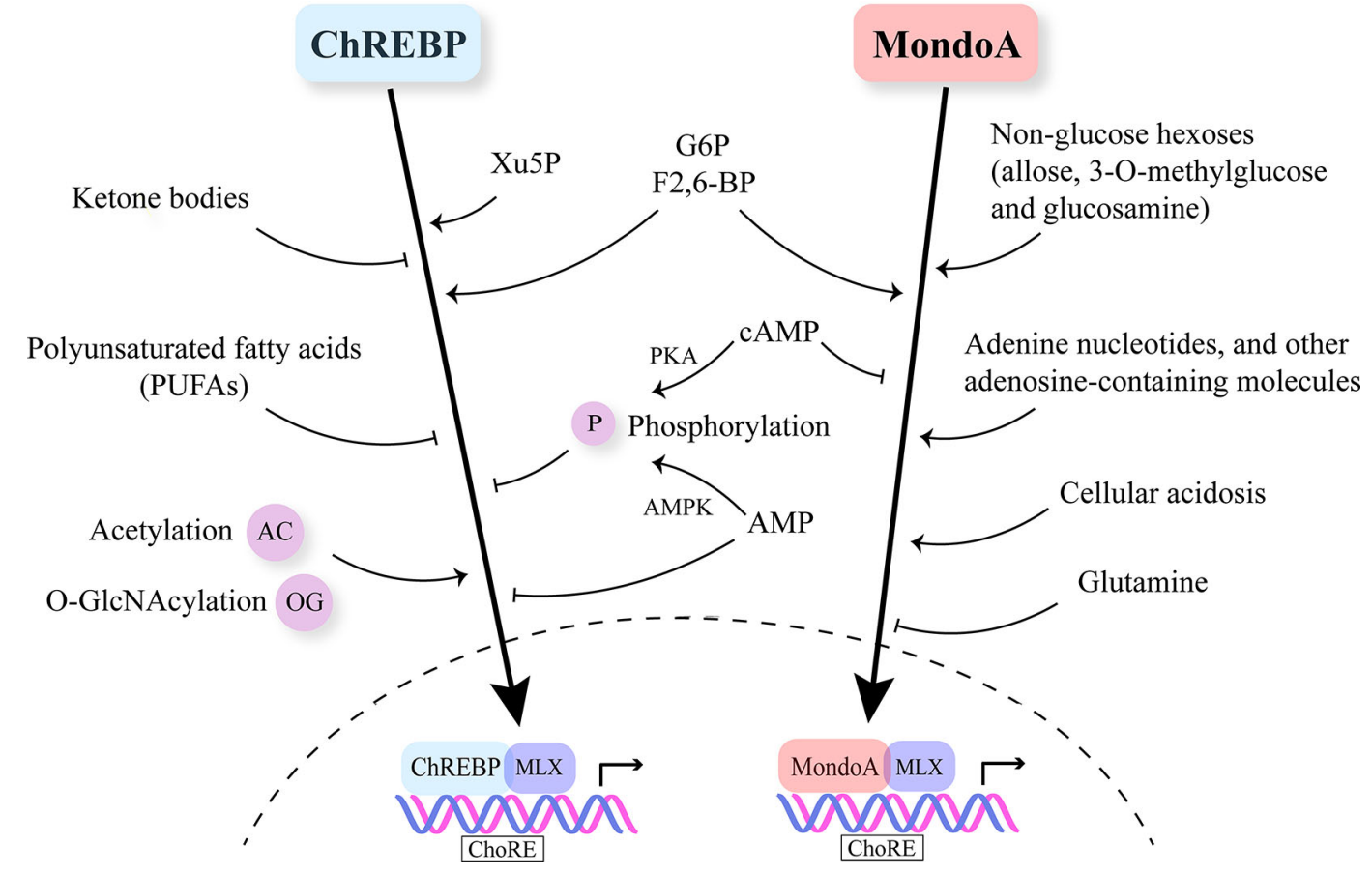

FIGURE 2 | Nutrient-sensing and regulation of Mondo family. Mondo family transcription factors sense multiple nutrients. G6P, F2,6-BP and Xu5P are considered the major metabolites through which glucose stimulates ChREBP and MondoA nuclear translocation and transcriptional activity. Ketone bodies and polyunsaturated fatty acids (PUFAs) are reported to inhibit ChREBP activity. MondoA activators include non-glucose hexoses, adenosine-containing molecules and cellular acidosis. Glutamine represses MondoA transcriptional activity. Post-transcriptional modifications (PTMs) including phosphorylation, acetylation and O-GlcNAcylation also play a role in regulating Mondo family, especially ChREBP. cAMP is a common inhibitor of ChREBP and MondoA. cAMP acts through PKA (protein kinase A) to promote the phosphorylation of ChREBP. Increased AMP levels lead to both retention of ChREBP in the cytosol and AMPK-induced phosphorylation of ChREBP, thus inhibiting ChREBP activity. 


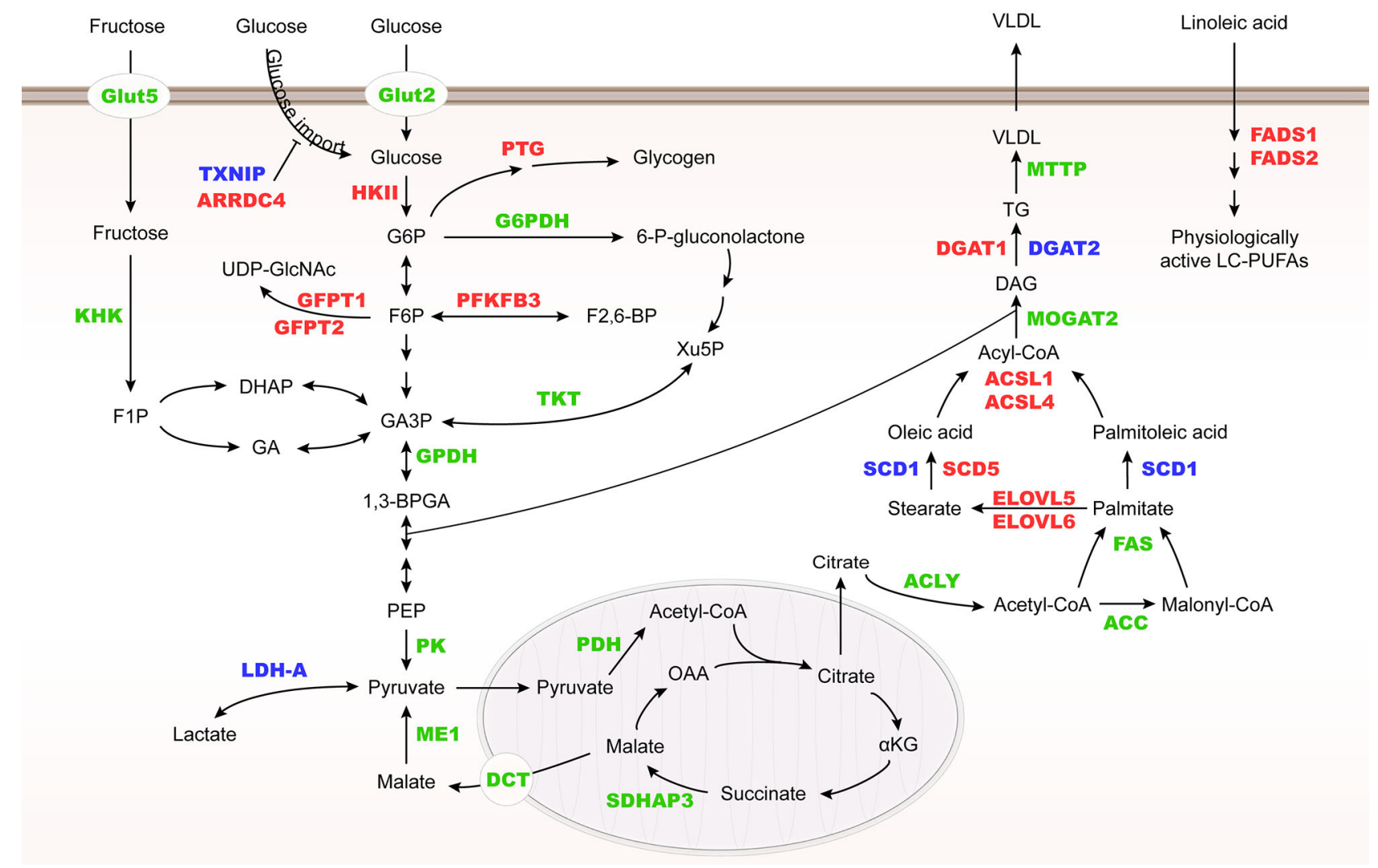

FIGURE 3 | Metabolic genes regulated by Mondo family. Metabolic genes regulated by MondoA and ChREBP at the transcriptional level are herein summarized. They are involved in glucose and fructose uptake, glycolysis, fructose metabolism, glycogenesis, hexosamine biosynthesis pathway (HBP), pentose phosphate pathway (PPP), and lipogenesis. MondoA targets are highlighted in red, ChREBP targets in green, and their common targets are in blue. HK II, hexokinase II; PTG, glycogen targeting protein; G6PDH, glucose-6-phosphate dehydrogenase; KHK, fructokinase; GFPT, glutamine:fructose-6-phosphate aminotransferase; PFKFB3, 6phosphofructo-2-kinase/fructose-2,6-biphosphatase 3; TKT, transketolase; PK, pyruvate kinase; PDH, pyruvate dehydrogenase; SDHAP3, succinate dehydrogenase complex flavoprotein subunit A pseudogene 3; DCT, C4-dicarboxylate transport protein; ME1, malic enzyme; MOGAT2, monoacylglycerol O-acyltransferase 2; DGAT, diacylglycerol acyltransferase.

cells (52). By shuttling between mitochondria and nucleus, MondoA bridges cytoplasmic nutrient level to transcriptional adaptations. MondoA localizes to the outer mitochondrial membrane under basal conditions, and accumulates in the nucleus in response to nutrient signals such as high glucose $(52,53)$. In addition to nuclear accumulation, glucose triggers MondoA-MLX binding to target promoters, and activates gene expression through recruitment of histone $\mathrm{H} 3$ acetyltransferase as coactivators (54).

Similar to ChREBP, MondoA senses levels of G6P and F2,6BP $(52,55)$ (Figure 2). Meanwhile, MondoA is responsive to non-glucose hexoses including allose and glucosamine (56). Intriguingly, glutamine recruits a histone deacetylasedependent corepressor to MondoA, turning MondoA-MLX into a transcriptional repressor. Moreover, cellular acidosis drives MondoA transcriptional activity since low $\mathrm{pH}$ promotes the production of mitochondrial ATP, with which mitochondriabound hexokinase generates G6P from cytoplasmic glucose (57). This finding has justified the special localization of MondoA-
MLX and unraveled the mechanisms underlying the activation of MondoA by lactic acidosis. Other molecules reported to be sensed by MondoA include adenine nucleotides and other adenosine-containing molecules $(58,59)$. Furthermore, mTOR (mammalian target of rapamycin), another key nutrient sensor, interacts with MondoA with a suppressive effect on its transcriptional activity (60). Nonetheless, so far there is no report on fatty acids or amino acids regulating MondoA level or activity.

Different from ChREBP, MondoA is predominantly expressed in skeletal muscle and immune cells (12), and our unpublished data. MondoA-deficient mice show enhanced glycolytic rates probably because loss of MondoA in skeletal muscle increases glucose uptake (43) (Table 1). In response to glucose and fructose, MondoA activates transcription of thioredoxin interacting protein (TXNIP) and arrestin domaincontaining 4 (ARRDC4), which inhibits glucose uptake (Figure 3) $(56,61)$. TXNIP, a dynamic sensor that modulates the energy demand of cells, plays a crucial role in the homeostasis of glucose. 
TABLE 1 | Roles of Mondo family on body weight, hepatic steatosis and insulin sensitivity according to mouse models, depending on nutritional status, genetic background and drug administration.

\begin{tabular}{|c|c|c|c|c|c|c|c|}
\hline $\begin{array}{l}\text { Mondo } \\
\text { family }\end{array}$ & Context & Modulation in Mouse Models & $\begin{array}{c}\text { Body } \\
\text { Weight }\end{array}$ & $\begin{array}{c}\text { Fat } \\
\text { Mass }\end{array}$ & $\begin{array}{l}\text { Hepatic } \\
\text { Steatosis }\end{array}$ & $\begin{array}{l}\text { Insulin } \\
\text { Sensitivity }\end{array}$ & Reference \\
\hline \multirow[t]{16}{*}{ ChREBP } & \multirow[t]{6}{*}{ Standard diet } & Global knockout & $=$ & $\searrow$ & $=$ & $\searrow$ & $(32,33)$ \\
\hline & & Liver-specific knockout & $=$ & $\searrow$ & $=$ & $\searrow$ & $(34)$ \\
\hline & & Liver-specific overexpression & $=$ & $\searrow$ & $\nearrow$ & $=$ & (35) \\
\hline & & AT-specific knockout & $=$ & $=$ & $\searrow$ & $\searrow$ & (36) \\
\hline & & AT-specific overexpression & $\searrow$ & $\searrow$ & $=$ & $=$ & (37) \\
\hline & & Pancreatic $\beta$-cell-specific overexpression & $\searrow$ & ND & ND & $\searrow$ & (38) \\
\hline & \multirow{6}{*}{$\begin{array}{l}\text { Standard diet in ob/ob mice } \\
\text { background } \\
\text { High-fat diet }\end{array}$} & Global knockout & $\searrow$ & $\searrow$ & $\searrow$ & $\nearrow$ & (33) \\
\hline & & Liver-specific knockdown & $\searrow$ & $\searrow$ & $\searrow$ & $\nearrow$ & (39) \\
\hline & & Liver-specific knockout & $=$ & $=$ & $=$ & $\searrow$ & (34) \\
\hline & & Liver-specific overexpression & $=$ & $\searrow$ & $\nearrow$ & $\nearrow$ & (35) \\
\hline & & AT-specific knockout & $=$ & $=$ & $=$ & $\searrow$ & (36) \\
\hline & & AT-specific overexpression & $\searrow$ & $\searrow$ & $\searrow$ & $\nearrow$ & (37) \\
\hline & Western diet & Global knockout & $\searrow$ & $\searrow$ & $\searrow$ & ND & $(40)$ \\
\hline & High-carbohydrate diet & Liver-specific knockout & $\searrow$ & $\searrow$ & $\searrow$ & $\searrow$ & (34) \\
\hline & \multirow{2}{*}{ HFrD } & Global knockout & $\searrow$ & ND & $\nearrow$ & ND & (41) \\
\hline & & Liver-specific knockout & $\searrow$ & $\searrow$ & $=$ & $\nearrow$ & (42) \\
\hline \multirow[t]{3}{*}{ MondoA } & \multirow[t]{2}{*}{ Standard diet } & Global knockout & $=$ & ND & ND & $=$ & (43) \\
\hline & & Muscle-specific knockout & $=$ & ND & ND & $=$ & $(44)$ \\
\hline & High-fat diet & Muscle-specific knockout & $=$ & ND & ND & $\nearrow$ & (44) \\
\hline $\begin{array}{l}\text { MondoA } \\
\text { /ChREBP }\end{array}$ & High-fat diet & $\begin{array}{l}\text { Administration of a compound (SBI-993) that deactivates } \\
\text { MondoA/ChREBP signaling }\end{array}$ & $\searrow$ & ND & $\searrow$ & $\nearrow$ & $(45)$ \\
\hline
\end{tabular}

"=" means not changed. ND, not determined. The upward and downward arrows indicate an increase and decrease in the level, respectively.

As a direct and glucose-responsive target of MondoA, TXNIP is upregulated when G6P level increases and concomitantly restricts glucose absorption, thus providing a negative feedback loop to prevent energy overload. Mechanisms underlying inhibition of glucose uptake regulated by TXNIP include the suppression of glucose transporter (GLUT) expression, GLUT vesicle transport and insulin signaling (44, 62-64). Moreover, MondoA enhances glycogen synthesis by activating the transcription of phosphoprotein phosphatase 1 regulatory subunit $3 \mathrm{~A}$ (PPP1R3A), phosphoprotein phosphatase 1 regulatory subunit 3B (PPP1R3B) and genes encoding the glycogen targeting subunits of protein phosphatase 1 (PP1) for promoting glycogen synthesis (65, 66). Muscle-specific MondoA knockout decreases glycogen level in the skeletal muscle of mice (62) (Table 1). Hence, under physiological conditions, glucose homeostasis is maintained by the downstream effects of MondoA activation.

In addition to glucose metabolism, MondoA diverts nutrients to lipid metabolic pathways, including fatty acid thioesterification [acyl-CoA synthetase 1, 4 (ACSL1, 4)], desaturation [fatty acid desaturase 1,2 (FADS1, 2), SCD1, 5], elongation [elongation of very long chain fatty acids protein 5, 6 (ELOVL5, 6)], and triglyceride synthesis [diacylglycerol acyltransferase 1, 2 (DGAT1, 2)] (44) (Figure 3). Taken together, as a nutrient-regulated transcription factor, MondoA not only decreases glucose import but also diverts nutrients to storage in skeletal muscle. Although various posttranslational modifications of ChREBP have been revealed to regulate its activity in different conditions (67-69), there is so far no report on how MondoA is posttranslationally modified. Therefore, further mechanistic studies are needed to elucidate the interacting protein network of MondoA in response to nutrient level alterations.

\section{THE ROLE OF MONDOA AND CHREBP IN OBESITY}

\section{ChREBP: From White to Brown and Beige Adipocytes}

Obesity is the excessive accumulation of fat caused by imbalance between energy intake and consumption. It is the major risk factor for many metabolic disorders such as type 2 diabetes, fatty liver and cardiovascular diseases (70). Regarded as a crucial target for the prevention and treatment of obesity, the adipose tissue consists of white adipocytes which store energy, and brown and beige adipocytes which consume energy and produce heat (71). Inducing beige adipocytes from white adipose tissues (WAT) is known as browning, a process which improves glucose metabolism and insulin sensitivity (11). Various transcription and endocrine factors participate in this process by directly or indirectly stimulating UCP1 expression in adipose tissues, including peroxisome proliferator-activated receptor $\gamma$ $(\operatorname{PPAR} \gamma), \operatorname{PPAR} \gamma$ coactivator- $1 \alpha$ (PGC- $1 \alpha)$, silent information regulator type 1 (SIRT1) and FGF21 (72). The activation of brown and beige adipocytes is considered to be an attractive therapeutic strategy for obesity and its comorbidities. Brown and beige adipocytes serve as a sink for excessive nutrients by promoting energy expenditure in mitochondria (73).

ChREBP promotes lipogenesis in adipose tissues $(36,74)$. For high-carbohydrate diets, excessive fructose and glucose are converted to fatty acids, in which a series of enzymes including ACLY, ACC and FAS participate (75). The predominant destiny of the newly synthesized fatty acids is to become triglycerides for storage, which helps to maintain energy homeostasis (76). Adipocyte de novo lipogenesis is also involved in the regulation of systemic insulin sensitivity and thermogenesis, both of which 
play key roles in mediating metabolic adaptations $(21,77)$. Overexpression of a constitutively active ChREBP isoform (caChREBP) in adipose tissues leads to an increase in expression of key enzymes involved in de novo lipogenesis (37). Conversely, adipocytes lacking ChREBP display impaired sucrose-induced lipogenesis (36). In WAT, Glut4-mediated glucose uptake induces ChREBP expression and activates the de novo lipogenic pathway. In Glut4 knockout mice, ChREBP expression in adipose tissues decreases by $50 \%$. It is noteworthy that Glut4-mediated changes in glucose flux have a stronger effect on the transcriptional expression of ChREBP $\beta$ than ChREBP $\alpha$ in WAT (16). ChREBP activity in adipocytes depends on ACLY, one of its transcriptional targets. In the absence of ACLY, the expression of both ChREBP and its target genes is significantly suppressed. Consequently, ACLY and ChREBP form a positive feedback loop in adipocytes to foster dietary carbohydrates uptake, fatty acid synthesis and storage of lipids (78). Moreover, specific ablation of Rictor, the essential subunit of the mechanistic target of rapamycin complex 2 (mTORC2) in mature adipocytes reduces ChREBP $\beta$ expression and downregulates de novo lipogenesis in WAT and brown adipose tissue (BAT) (79). In mature brown adipocytes, AKT2, which can be phosphorylated by $\mathrm{mTORC} 2$, is required for lipogenesis driven by ChREBP activation $(21,80)$.

To date, the role of ChREBP in regulating thermogenic adipocyte function has been indicated in a growing body of literatures (Figure 4). Reduced brown fat mass and hypothermia in response to excess energy intake are observed in ChREBPdeficient mice (32). ChREBP $\beta$ and UCP1 expression levels positively correlate in human BAT, suggesting that ChREBP $\beta$ expression might indicate brown adipocyte activity (21). Under chronic cold exposure, specific impairment of ChREBP-driven lipogenesis in BAT promotes beige adipocyte development, which is probably a compensatory response (21). Moreover, studies in adipocytes exposed to high glucose show that ChREBP is a critical mediator in triiodothyronine-induced upregulation of UCP1 expression in brown adipocytes (81). However, there is no significant binding of ChREBP protein to the UCP1 promoter, indicating that ChREBP regulates UCP1 transcription indirectly (82), which awaits further study. In mice fed a chronic high sucrose diet, expression of UCP1 in BAT is significantly increased compared with controls, which is probably due to the activated ChREBP-FGF21 axis (83). Moreover, overexpression of constitutively active ChREBP in adipocytes induces PPAR $\gamma$ activity and upregulates its thermogenesis-related target genes including UCP1 that promotes browning of WAT, while depletion of endogenous ChREBP in adipocytes has reciprocal effects (84). Furthermore, adenoviral overexpression of ChREBP in mice increases mRNA level of white adipose tissue UCP1 with increased plasma FGF21 level (46). After ChREBP $\beta$ is identified, it is important to consider the functional difference between the two isoforms of ChREBP. Of note, overexpression of ChREBP $\beta$ in brown adipocytes leads to impaired BAT thermogenesis and WAT browning, reflecting the role of ChREBP $\beta$ as a feedback regulator upon cold exposure (82). Moreover, given the expression of ChREBP in metabolic organs and macrophages, global gain-of-function or loss-of-function mouse models of ChREBP may not be ideal for analyzing the role of ChREBP in adipose tissues. Additional studies will be needed to develop a full picture of the specific role and mechanism of the two isoforms of ChREBP in adipocyte thermogenesis.

\section{MondoA: Inter-Organ Metabolic Crosstalk}

MondoA is expressed predominantly in skeletal muscle which makes up $\sim 40 \%$ of body weight and is responsible for $\sim 80 \%$ of glucose uptake $(62,85)$. Therefore, as a transcriptional factor

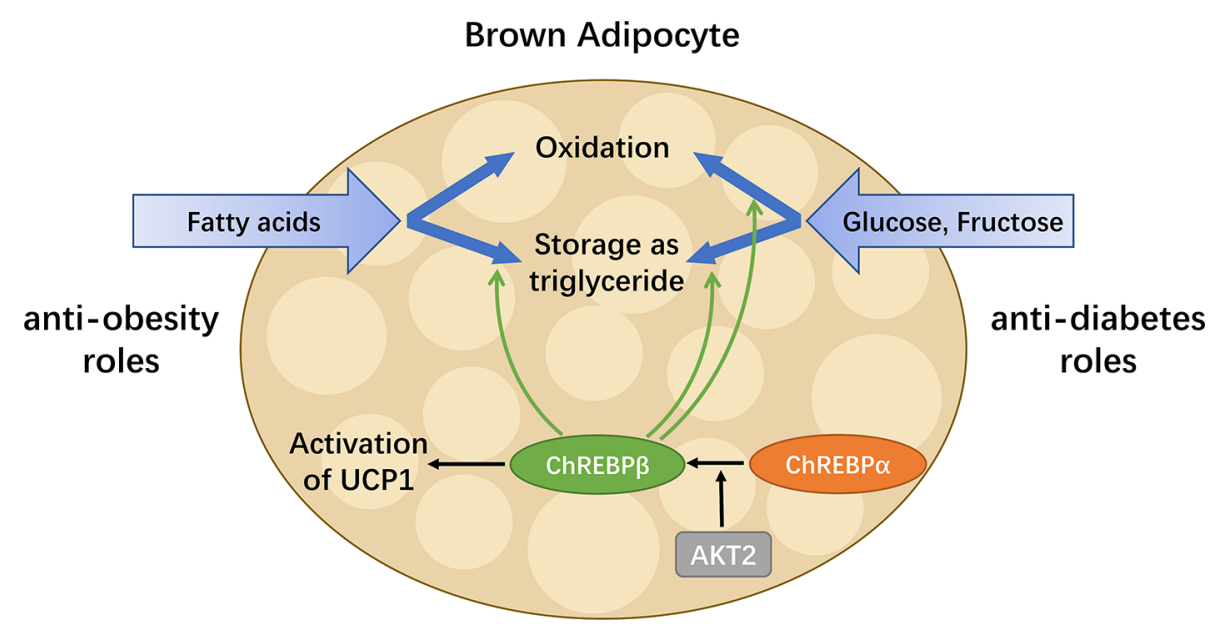

FIGURE 4 | ChREBP in BAT combats metabolic diseases. BAT has enormous promise for treating metabolic diseases including obesity and diabetes, as it is capable of taking up glucose, fructose and fatty acids, as well as oxidizing or storing them afterwards. ChREBP $\beta$, a truncated isoform of ChREBP, is most highly expressed in BAT and induced by the activation of the canonical isoform ChREBP $\alpha$. In addition to regulating key enzymes involved in metabolism pathways of glucose, fructose and fatty acids, ChREBP also mediates the activation of UCP1 in brown adipocytes. Thus, in BAT, ChREBP plays both anti-obesity and antidiabetes roles by increasing energy expenditure, reducing circulating glucose and improving insulin sensitivity. 
required for maintaining body homeostasis, MondoA plays a crucial part in inter-organ metabolic crosstalk.

In the context of chronic energy overload, MondoA is activated by glucose and fructose, which leads to the upregulation of TXNIP and ARRDC4, and concomitantly the impairment of glucose uptake in the skeletal muscle via suppression of insulin action $(44,45,55)$. Additionally, the chronic activation of MondoA in skeletal muscle results in lipotoxicity, namely deleterious effects of ectopic triglyceride accumulation $(44,45,55,86)$. Therefore, MondoA activation results in myocellular insulin resistance and lipid accumulation (44), serving as an intriguing supplement to the well-known insulin resistance based on triglyceride accumulation (87). These defects, along with hyperglycemia and hyperlipidemia, contribute to obesity-induced type 2 diabetes (T2D) (88).

In obesity, pancreatic $\beta$ cells adaptively produce more insulin to maintain blood glucose level, resulting in an amplified burden on $\beta$ cells. Intriguingly, MondoA serves as a significant glucoseresponsive transcription factor in $\beta$ cells (89). Under high glucose conditions, MondoA shuttles to the nucleus to induce its targets TXNIP and ARRDC4 in $\beta$ cells. TXNIP is a major factor promoting $\beta$ cell apoptosis (89-93). Hence, impaired $\beta$ cells might lead to progressive dysfunction of pancreas and even loss of its ability to produce and secrete insulin $(94,95)$.

All facts mentioned above will lead to an inter-organ vicious cycle of nutrient disposal and metabolism. The ideal solution is to limit energy intake while increasing the aerobic oxidation of fat in skeletal muscle by exercising (88). Therefore, to get out of the vicious cycle and regain the virtuous cycle, it is worthwhile to treat MondoA in skeletal muscle as a therapeutic target for obesity and insulin resistance.

\section{MONDO FAMILY AS A TARGET FOR METABOLIC DISORDERS}

In view of the central role of Mondo family in regulating energy homeostasis, the possibility to target MondoA or ChREBP in metabolic disorders has been explored.

As MondoA downregulates insulin sensitivity and promotes lipid storage in skeletal muscle $(44,45)$, it could be a promising therapeutic target for insulin resistance and lipotoxicity. For dietinduced obesity, muscle triglyceride accumulation and insulin resistance are partially relieved in muscle-specific MondoA knockout mice (44). MondoA deletion increases muscle glucose uptake and glycolytic capacity, resulting in enhanced sprint capacity (43). Moreover, SBI-477, a potent inhibitor of MondoA, alleviates muscle triglyceride levels and hepatic steatosis, thereby improving glucose tolerance in mice on a high-fat diet (45). However, the significant role of MondoA in skeletal muscle development has been recently revealed in mice (62). Therefore, in the development of MondoA as a novel therapeutic target, the timing for treatment is critical and the risk of interfering with normal myogenesis needs to be avoided.

Reducing ChREBP activity is considered as a promising target in the treatment of obesity according to studies utilizing $o b / o b$ and ChREBP double knockout mice (33). Of note, ChREBP plays an important role in promoting white adipocyte browning (81). Therefore, ChREBP in brown and beige adipocytes can be regarded as a treatment option for obesity. In consideration of the well-established role of brown and beige adipocytes in counteracting obesity, we await further research in this respect.

\section{SUMMARY}

Mondo family transcription factors are critical for metabolic homeostasis, as they sense multiple nutrient molecules and regulate metabolic enzyme genes transcriptionally. MondoA limits glucose uptake and glycolysis mostly in skeletal muscle and immune cells, while ChREBP promotes de novo lipogenesis in liver and adipose tissue. In pathological states of nutrient overload, MondoA could interfere with insulin signaling, while adipose ChREBP is linked to systemic insulin sensitivity and its role extends from white to brown and beige adipose tissues. The role of ChREBP in browning of white adipocytes is especially worth further exploration. Targeting MondoA and ChREBP to counteract obesity and related diseases is an appealing strategy that requires further investigations. As the manipulation of Mondo family in different organs and tissues could yield distinct systemic metabolic consequences, future studies should be conducted using more specific and rigorous models in order to clarify the beneficial or deleterious effects of Mondo family in different contexts. Meanwhile, before the therapeutic approaches could be developed, it is noteworthy that MondoA and ChREBP could be involved in normal myogenesis and adipogenesis. Moreover, under certain circumstances, the target genes and metabolic pathways of MondoA and ChREBP are overlapping. In this regard, in the knockout phenotype of one of the two transcription factors, whether the other acts in a compensatory way requires special attention. Furthermore, increasing studies reveal the involvement of Mondo family in critical signaling pathways, which awaits mechanistic investigations to expand our understanding of the action and regulation of these transcription factors.

\section{AUTHOR CONTRIBUTIONS}

XT designed and revised the manuscript. HK, YL, SW, and YZ wrote the manuscript, made the figures and the table. All authors contributed to the article and approved the submitted version.

\section{FUNDING}

This work was supported by grants from the 14th Undergraduate Training Program for Innovation of Shanghai Jiao Tong University School of Medicine (1420Y002), from National Natural Science Foundation of China (81672322, 81972210 and 92057117); the Shanghai Municipal Science and Technology Major Project (19JC1410200); National Key R\&D Program of China (2019YFA0906100); National Key Research and Development Program of China (No. 2016YFC1304800); Shanghai Jiao Tong 
University School of Medicine and Innovative research team of high-level local universities in Shanghai (SSMU-ZDCX20180400); The Program for Professor of Special Appointment (Eastern Scholar) at Shanghai Institutions of Higher Learning and Construction; Plan of Laboratory Technical Team in Shanghai Universities (SYjdyx19019).

\section{REFERENCES}

1. GBD 2017 Risk Factor Collaborators. Global, regional, and national comparative risk assessment of 84 behavioural, environmental and occupational, and metabolic risks or clusters of risks for 195 countries and territories, 1990-2017: a systematic analysis for the Global Burden of Disease Study 2017. Lancet (2018) 392:1923-94. doi: 10.1016/s0140-6736(18)32225-6

2. Ludwig DS, Peterson KE, Gortmaker SL. Relation between consumption of sugar-sweetened drinks and childhood obesity: a prospective, observational analysis. Lancet (2001) 357:505-8. doi: 10.1016/s0140-6736(00)04041-1

3. Schulze MB, Manson JE, Ludwig DS, Colditz GA, Stampfer MJ, Willett WC, et al. Sugar-sweetened beverages, weight gain, and incidence of type 2 diabetes in young and middle-aged women. Jama (2004) 292:927-34. doi: 10.1001/ jama.292.8.927

4. Sievenpiper JL, de Souza RJ, Mirrahimi A, Yu ME, Carleton AJ, Beyene J, et al. Effect of fructose on body weight in controlled feeding trials: a systematic review and meta-analysis. Ann Intern Med (2012) 156:291-304. doi: 10.7326/ 0003-4819-156-4-201202210-00007

5. Harms M, Seale P. Brown and beige fat: development, function and therapeutic potential. Nat Med (2013) 19:1252-63. doi: 10.1038/nm.3361

6. Cederberg A, Grønning LM, Ahrén B, Taskén K, Carlsson P, Enerbäck S. FOXC2 is a winged helix gene that counteracts obesity, hypertriglyceridemia, and diet-induced insulin resistance. Cell (2001) 106:563-73. doi: 10.1016/ s0092-8674(01)00474-3

7. Kopecky J, Clarke G, Enerbäck S, Spiegelman B, Kozak LP. Expression of the mitochondrial uncoupling protein gene from the aP2 gene promoter prevents genetic obesity. J Clin Invest (1995) 96:2914-23. doi: 10.1172/jci118363

8. Seale P, Conroe HM, Estall J, Kajimura S, Frontini A, Ishibashi J, et al. Prdm16 determines the thermogenic program of subcutaneous white adipose tissue in mice. J Clin Invest (2011) 121:96-105. doi: 10.1172/jci44271

9. Ortega-Molina A, Efeyan A, Lopez-Guadamillas E, Muñoz-Martin M, Gómez-López $\mathrm{G}$, Cañamero $\mathrm{M}$, et al. Pten positively regulates brown adipose function, energy expenditure, and longevity. Cell Metab (2012) 15:382-94. doi: 10.1016/j.cmet.2012.02.001

10. Vegiopoulos A, Müller-Decker K, Strzoda D, Schmitt I, Chichelnitskiy E, Ostertag A, et al. Cyclooxygenase-2 controls energy homeostasis in mice by de novo recruitment of brown adipocytes. Science (2010) 328:1158-61. doi: 10.1126/science.1186034

11. Lee P, Smith S, Linderman J, Courville AB, Brychta RJ, Dieckmann W, et al. Temperature-acclimated brown adipose tissue modulates insulin sensitivity in humans. Diabetes (2014) 63:3686-98. doi: 10.2337/db14-0513

12. Billin AN, Eilers AL, Coulter KL, Logan JS, Ayer DE. MondoA, a novel basic helix-loop-helix-leucine zipper transcriptional activator that constitutes a positive branch of a max-like network. Mol Cell Biol (2000) 20:8845-54. doi: $10.1128 / \mathrm{mcb} .20 .23 .8845-8854.2000$

13. Yamashita H, Takenoshita M, Sakurai M, Bruick RK, Henzel WJ, Shillinglaw W, et al. A glucose-responsive transcription factor that regulates carbohydrate metabolism in the liver. Proc Natl Acad Sci USA (2001) 98:9116-21. doi: 10.1073/pnas.161284298

14. Stoeckman AK, Ma L, Towle HC. Mlx is the functional heteromeric partner of the carbohydrate response element-binding protein in glucose regulation of lipogenic enzyme genes. J Biol Chem (2004) 279:15662-9. doi: 10.1074/ jbc.M311301200

15. Li MV, Chang B, Imamura M, Poungvarin N, Chan L. Glucose-dependent transcriptional regulation by an evolutionarily conserved glucose-sensing module. Diabetes (2006) 55:1179-89. doi: 10.2337/db05-0822

16. Herman MA, Peroni OD, Villoria J, Schön MR, Abumrad NA, Blüher M, et al. A novel ChREBP isoform in adipose tissue regulates systemic glucose metabolism. Nature (2012) 484:333-8. doi: 10.1038/nature10986

\section{ACKNOWLEDGMENTS}

We appreciate the useful suggestions from the members in the Tong laboratory. We apologize to those researchers whose work could not be cited or discussed in detail due to the space limitation.

17. Ge Q, Nakagawa T, Wynn RM, Chook YM, Miller BC, Uyeda K. Importinalpha protein binding to a nuclear localization signal of carbohydrate response element-binding protein (ChREBP). J Biol Chem (2011) 286:28119-27. doi: 10.1074/jbc.M111.237016

18. Eilers AL, Sundwall E, Lin M, Sullivan AA, Ayer DE. A novel heterodimerization domain, CRM1, and 14-3-3 control subcellular localization of the MondoA-Mlx heterocomplex. Mol Cell Biol (2002) 22:8514-26. doi: $10.1128 / \mathrm{mcb} .22 .24 .8514-8526.2002$

19. Sakiyama H, Wynn RM, Lee WR, Fukasawa M, Mizuguchi H, Gardner KH, et al. Regulation of nuclear import/export of carbohydrate response elementbinding protein (ChREBP): interaction of an alpha-helix of ChREBP with the 14-3-3 proteins and regulation by phosphorylation. J Biol Chem (2008) 283:24899-908. doi: 10.1074/jbc.M804308200

20. Li MV, Chen W, Poungvarin N, Imamura M, Chan L. Glucose-mediated transactivation of carbohydrate response element-binding protein requires cooperative actions from Mondo conserved regions and essential trans-acting factor 14-3-3. Mol Endocrinol (2008) 22:1658-72. doi: 10.1210/me.2007-0560

21. Sanchez-Gurmaches J, Tang Y, Jespersen NZ, Wallace M, Martinez Calejman C, Gujja S, et al. Brown Fat AKT2 Is a Cold-Induced Kinase that Stimulates ChREBP-Mediated De Novo Lipogenesis to Optimize Fuel Storage and Thermogenesis. Cell Metab (2018) 27:195-209. doi: 10.1016/j.cmet.2017. 10.008

22. Dentin R, Tomas-Cobos L, Foufelle F, Leopold J, Girard J, Postic C, et al. Glucose 6-phosphate, rather than xylulose 5-phosphate, is required for the activation of ChREBP in response to glucose in the liver. J Hepatol (2012) 56:199-209. doi: 10.1016/j.jhep.2011.07.019

23. McFerrin LG, Atchley WR. A novel N-terminal domain may dictate the glucose response of Mondo proteins. PloS One (2012) 7:e34803. doi: 10.1371/ journal.pone.0034803

24. Kabashima T, Kawaguchi T, Wadzinski BE, Uyeda K. Xylulose 5-phosphate mediates glucose-induced lipogenesis by xylulose 5-phosphate-activated protein phosphatase in rat liver. Proc Natl Acad Sci USA (2003) 100:510712. doi: $10.1073 /$ pnas. 0730817100

25. Arden C, Tudhope SJ, Petrie JL, Al-Oanzi ZH, Cullen KS, Lange AJ, et al. Fructose 2,6-bisphosphate is essential for glucose-regulated gene transcription of glucose-6-phosphatase and other ChREBP target genes in hepatocytes. Biochem J (2012) 443:111-23. doi: 10.1042/bj20111280

26. Sato S, Jung H, Nakagawa T, Pawlosky R, Takeshima T, Lee WR, et al. Metabolite Regulation of Nuclear Localization of Carbohydrate-response Element-binding Protein (ChREBP): ROLE OF AMP AS AN ALLOSTERIC INHIBITOR. J Biol Chem (2016) 291:10515-27. doi: 10.1074/jbc.M115.708982

27. Nakagawa T, Ge Q, Pawlosky R, Wynn RM, Veech RL, Uyeda K. Metabolite regulation of nucleo-cytosolic trafficking of carbohydrate response elementbinding protein (ChREBP): role of ketone bodies. J Biol Chem (2013) 288:28358-67. doi: 10.1074/jbc.M113.498550

28. Postic C, Dentin R, Denechaud P-D, Girard J. ChREBP, a transcriptional regulator of glucose and lipid metabolism. Annu Rev Nutr (2007) 27:179-92. doi: 10.1146/annurev.nutr.27.061406.093618

29. Ishii S, Iizuka K, Miller BC, Uyeda K. Carbohydrate response element binding protein directly promotes lipogenic enzyme gene transcription. Proc Natl Acad Sci USA (2004) 101:15597-602. doi: 10.1073/pnas.0405238101

30. Ma L, Robinson LN, Towle HC. ChREBP ${ }^{\star} \mathrm{Mlx}$ is the principal mediator of glucose-induced gene expression in the liver. J Biol Chem (2006) 281:2872130. doi: $10.1074 / j b c . M 601576200$

31. Niwa H, Iizuka K, Kato T, Wu W, Tsuchida H, Takao K, et al. ChREBP Rather Than SHP Regulates Hepatic VLDL Secretion. Nutrients (2018) 10:321. doi: 10.3390/nu10030321

32. Iizuka K, Bruick RK, Liang G, Horton JD, Uyeda K. Deficiency of carbohydrate response element-binding protein (ChREBP) reduces 
lipogenesis as well as glycolysis. Proc Natl Acad Sci USA (2004) 101:7281-6. doi: $10.1073 /$ pnas. 0401516101

33. Iizuka K, Miller B, Uyeda K. Deficiency of carbohydrate-activated transcription factor ChREBP prevents obesity and improves plasma glucose control in leptin-deficient (ob/ob) mice. Am J Physiol Endocrinol Metab (2006) 291:E358-64. doi: 10.1152/ajpendo.00027.2006

34. Jois T, Chen W, Howard V, Harvey R, Youngs K, Thalmann C, et al. Deletion of hepatic carbohydrate response element binding protein (ChREBP) impairs glucose homeostasis and hepatic insulin sensitivity in mice. Mol Metab (2017) 6:1381-94. doi: 10.1016/j.molmet.2017.07.006

35. Benhamed F, Denechaud PD, Lemoine M, Robichon C, Moldes M, BertrandMichel J, et al. The lipogenic transcription factor ChREBP dissociates hepatic steatosis from insulin resistance in mice and humans. J Clin Invest (2012) 122:2176-94. doi: 10.1172/JCI41636

36. Vijayakumar A, Aryal P, Wen J, Syed I, Vazirani RP, Moraes-Vieira PM, et al. Absence of Carbohydrate Response Element Binding Protein in Adipocytes Causes Systemic Insulin Resistance and Impairs Glucose Transport. Cell Rep (2017) 21:1021-35. doi: 10.1016/j.celrep.2017.09.091

37. Nuotio-Antar AM, Poungvarin N, Li M, Schupp M, Mohammad M, Gerard S, et al. FABP4-Cre Mediated Expression of Constitutively Active ChREBP Protects Against Obesity, Fatty Liver, and Insulin Resistance. Endocrinology (2015) 156:4020-32. doi: 10.1210/en.2015-1210

38. Poungvarin N, Lee JK, Yechoor VK, Li MV, Assavapokee T, Suksaranjit P, et al. Carbohydrate response element-binding protein (ChREBP) plays a pivotal role in beta cell glucotoxicity. Diabetologia (2012) 55:1783-96. doi: 10.1007/s00125-012-2506-4

39. Dentin R, Benhamed F, Hainault I, Fauveau V, Foufelle F, Dyck JR, et al. Liver-specific inhibition of ChREBP improves hepatic steatosis and insulin resistance in ob/ob mice. Diabetes (2006) 55:2159-70. doi: 10.2337/ db06-0200

40. Wu W, Tsuchida H, Kato T, Niwa H, Horikawa Y, Takeda J, et al. Fat and carbohydrate in western diet contribute differently to hepatic lipid accumulation. Biochem Biophys Res Commun (2015) 461:681-6. doi: $10.1016 /$ j.bbrc.2015.04.092

41. Zhang D, Tong X, VanDommelen K, Gupta N, Stamper K, Brady GF, et al. Lipogenic transcription factor ChREBP mediates fructose-induced metabolic adaptations to prevent hepatotoxicity. J Clin Invest (2017) 127:2855-67. doi: $10.1172 /$ jci89934

42. Kim M, Astapova II, Flier SN, Hannou SA, Doridot L, Sargsyan A, et al. Intestinal, but not hepatic, ChREBP is required for fructose tolerance. JCI Insight (2017) 2:e96703. doi: 10.1172/jci.insight.96703

43. Imamura M, Chang BH, Kohjima M, Li M, Hwang B, Taegtmeyer H, et al. MondoA deficiency enhances sprint performance in mice. Biochem J (2014) 464:35-48. doi: 10.1042/bj20140530

44. Ahn B, Wan S, Jaiswal N, Vega RB, Ayer DE, Titchenell PM, et al. MondoA drives muscle lipid accumulation and insulin resistance. JCI Insight (2019) 5: e129119. doi: 10.1172/jci.insight.129119

45. Ahn B, Soundarapandian MM, Sessions H, Peddibhotla S, Roth GP, Li JL, et al. MondoA coordinately regulates skeletal myocyte lipid homeostasis and insulin signaling. J Clin Invest (2016) 126:3567-79. doi: 10.1172/ jci87382

46. Iizuka K, Takao K, Kato T, Horikawa Y, Takeda J. ChREBP Reciprocally Regulates Liver and Plasma Triacylglycerol Levels in Different Manners. Nutrients (2018) 10:1699. doi: 10.3390/nu10111699

47. Linden AG, Li S, Choi HY, Fang F, Fukasawa M, Uyeda K, et al. Interplay between ChREBP and SREBP-1c coordinates postprandial glycolysis and lipogenesis in livers of mice. J Lipid Res (2018) 59:475-87. doi: 10.1194/ jlr.M081836

48. White PJ, McGarrah RW, Grimsrud PA, Tso SC, Yang WH, Haldeman JM, et al. and Lipid Metabolism via Regulation of ATP-Citrate Lyase. Cell Metab (2018) 27:1281-93.e7. doi: 10.1016/j.cmet.2018.04.015

49. Iizuka K, Takeda J, Horikawa Y. Glucose induces FGF21 mRNA expression through ChREBP activation in rat hepatocytes. FEBS Lett (2009) 583:2882-6. doi: 10.1016/j.febslet.2009.07.053

50. von Holstein-Rathlou S, BonDurant LD, Peltekian L, Naber MC, Yin TC, Claflin KE, et al. FGF21 Mediates Endocrine Control of Simple Sugar Intake and Sweet Taste Preference by the Liver. Cell Metab (2016) 23:335-43. doi: $10.1016 /$ j.cmet.2015.12.003
51. Fisher FM, Kim M, Doridot L, Cunniff JC, Parker TS, Levine DM, et al. A critical role for ChREBP-mediated FGF21 secretion in hepatic fructose metabolism. Mol Metab (2017) 6:14-21. doi: 10.1016/j.molmet.2016.11.008

52. Stoltzman CA, Peterson CW, Breen KT, Muoio DM, Billin AN, Ayer DE. Glucose sensing by MondoA : Mlx complexes: a role for hexokinases and direct regulation of thioredoxin-interacting protein expression. Proc Natl Acad Sci USA (2008) 105:6912-7. doi: 10.1073/pnas.0712199105

53. Sans CL, Satterwhite DJ, Stoltzman CA, Breen KT, Ayer DE. MondoA-Mlx heterodimers are candidate sensors of cellular energy status: mitochondrial localization and direct regulation of glycolysis. Mol Cell Biol (2006) 26:486371. doi: $10.1128 / \mathrm{mcb} .00657-05$

54. Peterson CW, Stoltzman CA, Sighinolfi MP, Han KS, Ayer DE. Glucose controls nuclear accumulation, promoter binding, and transcriptional activity of the MondoA-Mlx heterodimer. Mol Cell Biol (2010) 30:2887-95. doi: $10.1128 / \mathrm{mcb} .01613-09$

55. Petrie JL, Al-Oanzi ZH, Arden C, Tudhope SJ, Mann J, Kieswich J, et al. Glucose induces protein targeting to glycogen in hepatocytes by fructose 2,6bisphosphate-mediated recruitment of MondoA to the promoter. Mol Cell Biol (2013) 33:725-38. doi: 10.1128/mcb.01576-12

56. Stoltzman CA, Kaadige MR, Peterson CW, Ayer DE. MondoA senses nonglucose sugars: regulation of thioredoxin-interacting protein (TXNIP) and the hexose transport curb. J Biol Chem (2011) 286:38027-34. doi: 10.1074/ jbc.M111.275503

57. Wilde BR, Ye Z, Lim TY, Ayer DE. Cellular acidosis triggers human MondoA transcriptional activity by driving mitochondrial ATP production. Elife (2019) 8:e40199. doi: 10.7554/eLife.40199

58. Yu FX, Goh SR, Dai RP, Luo Y. Adenosine-containing molecules amplify glucose signaling and enhance txnip expression. Mol Endocrinol (2009) 23:932-42. doi: 10.1210/me.2008-0383

59. Han KS, Ayer DE. MondoA senses adenine nucleotides: transcriptional induction of thioredoxin-interacting protein. Biochem J (2013) 453:209-18. doi: 10.1042/bj20121126

60. Kaadige MR, Yang J, Wilde BR, Ayer DE. MondoA-Mlx transcriptional activity is limited by mTOR-MondoA interaction. Mol Cell Biol (2015) 35:101-10. doi: 10.1128/mcb.00636-14

61. Parikh H, Carlsson E, Chutkow WA, Johansson LE, Storgaard H, Poulsen P, et al. TXNIP regulates peripheral glucose metabolism in humans. PloS Med (2007) 4:e158. doi: 10.1371/journal.pmed.0040158

62. Ran H, Lu Y, Zhang Q, Hu Q, Zhao J, Wang K, et al. MondoA Is Required for Normal Myogenesis and Regulation of the Skeletal Muscle Glycogen Content in Mice. Diabetes Metab J (2020). doi: 10.4093/dmj.2019.0212

63. Mandala A, Das N, Bhattacharjee S, Mukherjee B, Mukhopadhyay S, Roy SS. Thioredoxin interacting protein mediates lipid-induced impairment of glucose uptake in skeletal muscle. Biochem Biophys Res Commun (2016) 479:933-9. doi: 10.1016/j.bbrc.2016.09.168

64. Waldhart AN, Dykstra H, Peck AS, Boguslawski EA, Madaj ZB, Wen J, et al. Phosphorylation of TXNIP by AKT Mediates Acute Influx of Glucose in Response to Insulin. Cell Rep (2017) 19:2005-13. doi: 10.1016/ j.celrep.2017.05.041

65. Delibegovic M, Armstrong CG, Dobbie L, Watt PW, Smith AJH, Cohen PTW. Disruption of the striated muscle glycogen targeting subunit PPP1R3A of protein phosphatase 1 leads to increased weight gain, fat deposition, and development of insulin resistance. Diabetes (2003) 52:596-604. doi: 10.2337/ diabetes.52.3.596

66. Montori-Grau M, Guitart M, Lerin C, Andreu AL, Newgard CB, GarcíaMartínez C, et al. Expression and glycogenic effect of glycogen-targeting protein phosphatase 1 regulatory subunit GL in cultured human muscle. Biochem J (2007) 405:107-13. doi: 10.1042/BJ20061572

67. Kawaguchi T, Takenoshita M, Kabashima T, Uyeda K. Glucose and cAMP regulate the L-type pyruvate kinase gene by phosphorylation/ dephosphorylation of the carbohydrate response element binding protein. Proc Natl Acad Sci USA (2001) 98:13710-5. doi: 10.1073/pnas.231370798

68. Bricambert J, Miranda J, Benhamed F, Girard J, Postic C, Dentin R. Saltinducible kinase 2 links transcriptional coactivator p300 phosphorylation to the prevention of ChREBP-dependent hepatic steatosis in mice. J Clin Invest (2010) 120:4316-31. doi: 10.1172/jci41624

69. Guinez C, Filhoulaud G, Rayah-Benhamed F, Marmier S, Dubuquoy C, Dentin R, et al. O-GlcNAcylation increases ChREBP protein content and 
transcriptional activity in the liver. Diabetes (2011) 60:1399-413. doi: 10.2337/ db10-0452

70. Carobbio S, Pellegrinelli V, Vidal-Puig A. Adipose Tissue Function and Expandability as Determinants of Lipotoxicity and the Metabolic Syndrome. Adv Exp Med Biol (2017) 960:161-96. doi: 10.1007/978-3-319-48382-5_7

71. Lynes MD, Tseng Y-H. Deciphering adipose tissue heterogeneity. Ann N Y Acad Sci (2018) 1411:5-20 doi: 10.1111/nyas.13398

72. Montanari T, Pošćić N, Colitti M. Factors involved in white-to-brown adipose tissue conversion and in thermogenesis: a review. Obes Rev (2017) 18:495513. doi: $10.1111 /$ obr. 12520

73. Ricquier D. UCP1, the mitochondrial uncoupling protein of brown adipocyte: A personal contribution and a historical perspective. Biochimie (2017) 134:38. doi: 10.1016/j.biochi.2016.10.018

74. Song Z, Xiaoli AM, Yang F. Regulation and Metabolic Significance of Lipogenesis in Adipose Tissues. Nutrients (2018) 10:1383. doi: 10.3390/ nu10101383

75. Ameer F, Scandiuzzi L, Hasnain S, Kalbacher H, Zaidi N. De novo lipogenesis in health and disease. Metabolism (2014) 63:895-902. doi: 10.1016/ j.metabol.2014.04.003

76. Lodhi IJ, Wei X, Semenkovich CF. Lipoexpediency: de novo lipogenesis as a metabolic signal transmitter. Trends Endocrinol Metab (2011) 22:1-8. doi: $10.1016 /$ j.tem.2010.09.002

77. Smith U, Kahn BB. Adipose tissue regulates insulin sensitivity: role of adipogenesis, de novo lipogenesis and novel lipids. J Intern Med (2016) 280:465-75. doi: 10.1111/joim.12540

78. Fernandez S, Viola JM, Torres A, Wallace M, Trefely S, Zhao S, et al. Adipocyte ACLY Facilitates Dietary Carbohydrate Handling to Maintain Metabolic Homeostasis in Females. Cell Rep (2019) 27:2772-84.e6. doi: 10.1016/j.celrep.2019.04.112

79. Tang Y, Wallace M, Sanchez-Gurmaches J, Hsiao WY, Li H, Lee PL, et al. Adipose tissue mTORC2 regulates ChREBP-driven de novo lipogenesis and hepatic glucose metabolism. Nat Commun (2016) 7:11365. doi: 10.1038/ ncomms11365

80. Sanchez-Gurmaches J, Martinez Calejman C, Jung SM, Li H, Guertin DA. Brown fat organogenesis and maintenance requires AKT1 and AKT2. Mol Metab (2019) 23:60-74. doi: 10.1016/j.molmet.2019.02.004

81. Katz LS, Xu S, Ge K, Scott DK, Gershengorn MC. T3 and Glucose Coordinately Stimulate ChREBP-Mediated Ucp1 Expression in Brown Adipocytes From Male Mice. Endocrinology (2018) 159:557-69. doi: 10.1210/en.2017-00579

82. Wei C, Ma X, Su K, Qi S, Zhu Y, Lin J, et al. ChREBP- $\beta$ regulates thermogenesis in brown adipose tissue. J Endocrinol (2020) 245:343-56. doi: 10.1530/JOE-19-0498

83. Maekawa R, Seino Y, Ogata H, Murase M, Iida A, Hosokawa K, et al. Chronic high-sucrose diet increases fibroblast growth factor 21 production and energy expenditure in mice. J Nutr Biochem (2017) 49:71-9. doi: 10.1016/ j.jnutbio.2017.07.010

84. Witte N, Muenzner M, Rietscher J, Knauer M, Heidenreich S, Nuotio-Antar AM, et al. The Glucose Sensor ChREBP Links De Novo Lipogenesis to PPARgamma Activity and Adipocyte Differentiation. Endocrinology (2015) 156:4008-19. doi: 10.1210/EN.2015-1209
85. DeFronzo RA, Gunnarsson R, Björkman O, Olsson M, Wahren J. Effects of insulin on peripheral and splanchnic glucose metabolism in noninsulindependent (type II) diabetes mellitus. J Clin Invest (1985) 76:149-55. doi: $10.1172 / \mathrm{JCI} 111938$

86. Sartor F, Jackson MJ, Squillace C, Shepherd A, Moore JP, Ayer DE, et al. Adaptive metabolic response to 4 weeks of sugar-sweetened beverage consumption in healthy, lightly active individuals and chronic high glucose availability in primary human myotubes. Eur J Nutr (2013) 52:937-48. doi: 10.1007/s00394-012-0401-x

87. Pan DA, Lillioja S, Kriketos AD, Milner MR, Baur LA, Bogardus C, et al. Skeletal muscle triglyceride levels are inversely related to insulin action. Diabetes (1997) 46:983-8. doi: 10.2337/diab.46.6.983

88. Simonson DC, Halperin F, Foster K, Vernon A, Goldfine AB. Clinical and Patient-Centered Outcomes in Obese Patients With Type 2 Diabetes 3 Years After Randomization to Roux-en-Y Gastric Bypass Surgery Versus Intensive Lifestyle Management: The SLIMM-T2D Study. Diabetes Care (2018) 41:6709. doi: $10.2337 / \mathrm{dc} 17-0487$

89. Richards P, Rachdi L, Oshima M, Marchetti P, Bugliani M, Armanet M, et al. MondoA Is an Essential Glucose-Responsive Transcription Factor in Human Pancreatic $\beta$-Cells. Diabetes (2018) 67:461-72. doi: 10.2337/db17-0595

90. Thielen L, Shalev A. Diabetes pathogenic mechanisms and potential new therapies based upon a novel target called TXNIP. Curr Opin Endocrinol Diabetes Obes (2018) 25:75-80. doi: 10.1097/MED.0000000000000391

91. Lerner AG, Upton J-P, Praveen PVK, Ghosh R, Nakagawa Y, Igbaria A, et al. IRE1 $\alpha$ induces thioredoxin-interacting protein to activate the NLRP3 inflammasome and promote programmed cell death under irremediable ER stress. Cell Metab (2012) 16:250-64. doi: 10.1016/j.cmet.2012.07.007

92. Chen J, Hui ST, Couto FM, Mungrue IN, Davis DB, Attie AD, et al. Thioredoxin-interacting protein deficiency induces Akt/Bcl-xL signaling and pancreatic beta-cell mass and protects against diabetes. FASEB J: Off Publ Fed Am Societies Exp Biol (2008) 22:3581-94. doi: 10.1096/fj.08-111690

93. Saxena G, Chen J, Shalev A. Intracellular shuttling and mitochondrial function of thioredoxin-interacting protein. J Biol Chem (2010) 285:39974005. doi: 10.1074/jbc.M109.034421

94. Boland BB, Rhodes CJ, Grimsby JS. The dynamic plasticity of insulin production in $\beta$-cells. Mol Metab (2017) 6:958-73. doi: 10.1016/ j.molmet.2017.04.010

95. Robertson RP. Chronic oxidative stress as a central mechanism for glucose toxicity in pancreatic islet beta cells in diabetes. J Biol Chem (2004) 279:423514. doi: $10.1074 / \mathrm{jbc}$.R400019200

Conflict of Interest: The authors declare that the research was conducted in the absence of any commercial or financial relationships that could be construed as a potential conflict of interest.

Copyright () $2021 \mathrm{Ke}, \mathrm{Luan}, \mathrm{Wu}, \mathrm{Zhu}$ and Tong. This is an open-access article distributed under the terms of the Creative Commons Attribution License (CC BY). The use, distribution or reproduction in other forums is permitted, provided the original author(s) and the copyright owner(s) are credited and that the original publication in this journal is cited, in accordance with accepted academic practice. No use, distribution or reproduction is permitted which does not comply with these terms. 